\title{
Symmetry-induced vortex patterns
}

Victor V. Moshchalkov, Laboratorium voor Vaste-Stoffysica en Magnetisme, Katholieke Unuversiteit Leuven, Celestijnenlaan 200 D, B-3001 Leuven, Belgium $F_{\text {ture superconductivity in the cuprates perovskites by Mueller }}^{\text {or about } 15 \text { years, that is since the discovery high tempera- }}$ and Bednorz, many breakthroughs have been reported, the last one being the observation of superconductivity in $\mathrm{MgB} 2$ around $40 \mathrm{~K}$. Perhaps less publicized are the spectacular progress made during this period in the fabrication of superconducting nanostructures and their regular arrays to understand new quantum effects. With respect to the phenomenology of superconductors described by the Ginzburg-Landau theory, a particular interesting question to address is the influence of sample size and geometry on the confinement of superconducting vortices. It is like the well known quantum mechanical problem of a particle in a box: different boxes having different quantum levels [1].

The fabrication of such nanostructures (including different regular pinning arrays) with sizes ranging from $1 \mu \mathrm{m}$ to $10 \mu \mathrm{m}$ can be made by photolithography and electron beam lithography, and down to $0.1 \mathrm{~nm}$ by single atom manipulation. This progress has strongly stimulated the experimental and theoretical studies of nanostructured systems, including superconductors. This is one of the main research topics in the framework of the ongoing European Science Foundation Programme "Vortex Matter in Superconductors at Extreme Scales and Conditions" -VORTEX [2]. In contrast to the conventional approach, which relied upon the search for new bulk materials each time a new specific combination of physical properties was required, the modern trend in condensed matter physics is to modify the properties of the same material through its nanostructuring and the optimization of the confinement potential and topology (concept of "quantum design").

One of the main objectives of the ESF Programme VORTEX is to investigate the role of the vortex confinement in the evolution of the properties of the vortex matter at extreme length scales and conditions. The main envisaged achievement of the program will be the (hopefully positive) answer to the following fundamental question: can the quantum design of the vortex confinement in superconductors result in the optimization of their critical currents and fields? By optimizing the vortex confinement, we shall be able to increase these two fundamental parameters of any given superconductor up to their theoretical limits, which is of course very important for applications.

Three main classes of nanoengineered superconducting materials are interesting to investigate in that respect: (i) individual nanoplaquettes of different geometry, (ii) clusters of them and (iii) large arrays. For individual nanoplaquettes a very straitforward fundamental question arises: to what extent are the properties of the confined vortices affected by the imposed discrete symmetry of the sample? This important question has been recently addressed by the two teams at the Katholieke Universiteit Leuven (Laboratories of Solid State Physics and Magnetism, and Quantum Chemistry) [3,4]. In disks and cylinders, for example, a big single whirl in the center ("giant vortex state") is compatible with the symmetry of the boundary conditions. In superconducting equilateral triangles, squares, etc, however, a very different situation arises. For example, it is quite easy to keep the imposed symmetry trying to put three vortices into a triangle or four into a square. But what about "less lucky" numbers, like two vortices in a triangle or three vortices in a square (see the Figure)? The latter is like "...pushing a triangular peg into a square hole" [5]. Seemingly the only possible solution of this puzzle is to "merge" single quantum vortices into a single "giant" vortex and to place it in the center. In the language of numbers it is like presenting the number of applied flux quanta through the sample $2=2+0$ instead of $2=$ $1+1$ for two vortices in triangle or, similarly, $3=3+0$ instead of $3=$ $1+1+1$ for three vortices in a square. But such a "merger" costs too much energy in triangles and squares, although is possible in a disc or a cylinder. If the formation of the giant vortex state is excluded, then it looks like there is no solution of the puzzle. Remarkably, spontaneous formation of the vortex - antivortex pair saves the whole scheme. Indeed, using the same symbolic language of numbers, we can claim that not only $2=2+0$ but also $2=$ $3-1$, meaning three vortices in three corners of a triangle and one
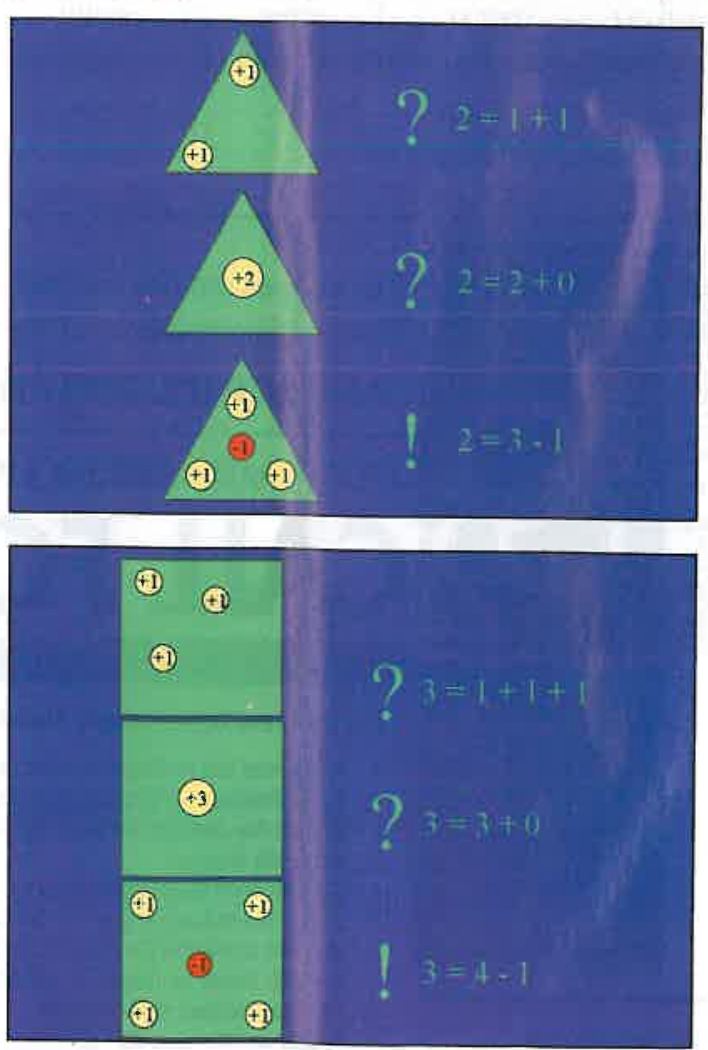

Schematic presentation of the vortex patterns in mesoscopic superconducting triangles and squares for the applied magnetic field generating two flux quanta through a triangle (top panel) or three flux quanta through a square (bottom panel). The spontaneous generation of the vortex-antivortex pair keeps the applied vorticity $(1-1=0)$ and at the same time solves the puzzle how to find the symmetry-consistent vortex configurations, corresponding to $2=3-1$ (triangle) and $3=4-1$ (square). 
antivortex in the center (fully symmetry consistent!) or, for a square, instead of $3=3+0$, we can use $3=4-1$, thus making it possible to put comfortably four vortices into four corners and one antivortex into the center. It looks like a "discovery" of negative numbers (antivortices, in our case) provides the necessary means for the vortex system to comply with the imposed boundary conditions. Note, that these symmetry-induced antivortices are qualitatively different from the ones related to the KosterlitzThouless transition in two-dimensional superconductors.

The first experimental evidence supporting the existence of the symmetry induced antivortices in mesoscopic superconducting triangles and squares has been found in measurements of the phase boundary defined by the field dependence of the superconducting critical temperature $\mathrm{Tc}(\mathrm{H})$. These measurements revealed a very good agreement between the boundary calculated from the linearised Ginzburg-Landau equation (LGLE) taking into account the formation of antivortices, and the experimental $\mathrm{Tc}(\mathrm{H})$ line $[3,4]$. More experimental work is under the way to use the vortex imaging techniques, such as scanning SQUID, Lorentz or Hall microscopes, to visualize directly the symmetry-induced vortex patterns. Theoretically, the effects of the non-linear terms in the GLE should be also considered in order to find out which vortex configurations survive when one moves deeper into the superconducting state.
These new ideas about the spontaneous formation of the vortex-antivortex pairs are applicable to other types of the symmetrically confined vortex matter, like superfluid helium rotated in a triangular or square vessel, or Bose-Einstein condensate confined in a trap with a well defined discrete symmetry and certain vorticity imposed by the rotated laser beam. Future experimental and theoretical efforts in this area will lead to a better understanding of the fundamental physics behind puzzling questions about interplay between the discrete symmetry of the sample and the vortex patterns consistent with it.

\section{References}

[1] V.V.Moshchalkov et al, Nature, 373, 319 (1995).

[2] http://www.fys.kuleuven.ac.be/vortex

[3] L.F.Chibotaru, A.Ceulemans, V.Bruyndoncx, V.V.Moshchalkov, Nature, 408, 833 (2000).

[4] L.F.Chibotaru, A.Ceulemans, V.Bruyndoncx, V.V.Moshchalkov, Phys. Rev. Lett., 86, 1321 (2001).

[5] A.T.Dorsey, Nature, 408, 833 (2000). 